\title{
Comparison of neuroglobin distribution and expression between the retina of the adult Bactrian camel, rabbits and sheep")
}

\author{
XIAOHUA DU, JAMES BLACKAR MAWOLO*, XIA LIU* \\ College of Veterinary Medicine, *College of Life Science and Technology, \\ Gansu Agricultural University, Lanzhou City, Gansu Province, People's Republic of China
}

Du X., Mawolo J. B., Liu X.

Comparison of neuroglobin distribution and expression between the retina of the adult Bactrian camel, rabbits and sheep

\section{Summary}

Neuroglobin (Ngb) is a kind of protein largely expressed in the brain and retina of mammals. Numerous studies have reported on Ngb expression and distribution in mammals but none have compared the expression in the adult Bactrian camel, rabbits, and sheep. The study examined the distribution and expression of Ngb between the retina of adult Bactrian camel, rabbits, and sheep and provides detailed insight on the morphology of these mammals' retinae. The immunohistochemical staining procedures were performed to detect Ngb distribution and its expression in the retinae of the adult Bactrian camel, rabbits, and sheep. The results showed that strong positive Ngb expression was found in all layers of the Bactrian camel except the outer nuclear layer, while in the rabbit retina, the strong positive expression was observed in the cortex of the optic nerve fiber layer, the retina cells layer, the network layer, the photoreceptor inner segment, and the pigment, while weak positive expression was shown in the retina of the kernel layer, outside the outer nuclear layer of the retina and the light receptor section. In the adult sheep retina, Ngb was solely expressed in the nerve fiber layer, inner and outer plexiform layer, optic nerve, inner and outer limiting membrane, and photoreceptor inner segment, while weak positive expression was shown in the ganglion cell layer and inner nuclear layer. There exist no Ngb positive expression in the photoreceptor outer segment, the outer nuclear layer, and retinal pigment epithelium of the adult sheep retina. The study documented that Ngb may have a significant function in the maintenance of retinal oxygen homeostasis and participation in the repair of light damage. The study also provided detailed references for Ngb physiological function and its relationship to extreme environmental conditions.

Keywords: Neuroglobin, retina, immunohistochemistry, Bactrian camel, rabbit and sheep

Neuroglobin $(\mathrm{Ngb})$ is a neuronal hemeprotein similar to myoglobin and hemoglobin and shares their capability for oxygen binding. Research has shown that $\mathrm{Ngb}$ expression in the retina is higher than other neural tissues, and its concentration is about 50-100 times (26). Yu et al. reported that in species with vascularized retina, oxygen is transported to the retina in combination with the choroidal vascular bed, which is located at the back of the retina and is the highest consumer of oxygen (7). Another study confirmed that the ocular tissues in the retina are unique and have the highest rates

1) The research project was funded by the National Natural Science Foundation of China (Grant No. 31760305), The Youth Foundation of Gansu Agricultural University (Grant No. GAU-QNDS-201501), and the National Natural Foundation of Gansu Province (Grant No. 17JR5RA142). The funders had no role in study design, data collection, and analysis, decision to publish, or preparation of the manuscript. of oxygen consumption (8). Scientists have studied the expression of $\mathrm{Ngb}$ in the retina of rodents and humans $(10,11)$ and reported significant references about these animals. The expression level of $\mathrm{Ngb}$ in the retina is much higher than in the brain tissue $(12,13)$, suggesting that there exists a close relationship between $\mathrm{Ngb}$ and retinal functions. Studies have shown that Ngb is involved in the elimination of reactive oxygen species (ROS) regulation which may play an important role in oxygen homeostasis (14). Further research conducted by Ostoji et al. reported that the expression of $\mathrm{Ngb}$ was solely found in the ganglion cell layer, inner nuclear layer, inner and outer plexiform layers, and retinal pigment epithelium but not in the glial, suggesting that $\mathrm{Ngb}$ could serve a neuroprotective role as scavengers of reactive oxygen species and developing therapeutic 
strategies for the treatment of hypoxia-related ocular diseases $(15,29)$. Despite all these results, there are no references for $\mathrm{Ngb}$ expression in the retina of the Bactrian camel, rabbits, and sheep. The Bactrian camel (Bactrian camel) is a domestic animal that lives in desert and semi-desert areas and is highly adaptable to the extreme natural environment of drought and heat while adult rabbits are small mammals in the family Leporidae and need a regulated environment to protect them against heat exhaustion or hypothermia. Sheep are ruminant (cud-chewing) mammals of the genus Ovis and live throughout the world, especially in mountainous areas. The current research examined $\mathrm{Ngb}$ expression in the retina of healthy adult Bactrian camels, rabbits, and sheep by employing immunohistochemical staining procedures. The research also documented a theoretical foundation for understanding the repair function of $\mathrm{Ngb}$ in the retina injury of mammals under extreme natural environment and supplied morphological data.

\section{Material and methods}

Animals and setting. The procedures used in this study were reviewed and approved by the Animal Ethics and Welfare Committee of Approval Number: Gansu Agricultural University (GAU-AEW-2019-0026).

A total of three (3) healthy adult camels (7 years), five (5) adult rabbits (6 years), and five (5) healthy adult sheep (6 years) were involved in the study. The healthy adult camels were purchased from the Zhangye prefecture-level areas in Wuwei, Gansu province, China while the rabbits and sheep used in this study were taken from Lanzhou city in Gansu Province, China were tested, and showed no sign of any disease. Specimens were collected in a morning and evening hour during July and collection was done within one week. Animals were retrieved one after another from their housing area and taken to the slaughterhouse. Zhangye prefecture is located in central Gansu along the Hexi Corridor, occupying $42,000 \mathrm{~km}^{2}(16,000 \mathrm{sq} \mathrm{mi})$. It takes up the entire breadth of the province, running from Inner Mongolia on the north to Qinghai on the south, but its urban core is at Ganzhou in the oasis formed by the Ruo or Hei River and the Lanzhou city is the capital and largest city of Gansu Province and is noted for its yellow river and situated in the temperate zone with a semi-arid climate during hot summer and extremely cold and dry winter. The average monthly temperature ranges from $-5.3^{\circ} \mathrm{C}\left(22.5^{\circ} \mathrm{F}\right)$ in January to $22.4^{\circ} \mathrm{C}\left(72.3^{\circ} \mathrm{F}\right)$ in July.

Samples extraction and preparation. Before been sacrificed, all animals were fed and rested for a while. The animals were sacrificed, the cornea was open with a sharp blade and the eyeballs were quickly removed and placed in Davidson's fixation solution ( $95 \%$ alcohol, $37 \%$ strong formalin, glacial acetic acid, distilled water ratio: $3: 2: 1: 2$ ) for 24 hours and stored at $4^{\circ} \mathrm{C}$. Later, the perfusion-fixed retinae were placed on disinfected glass slides, and normal sheep serum (ab7489, North America) was added to block the non-specific binding sides at room temperature for an hour in Phosphate-buffered saline (PBS, Beijing, China). These sections were incubated with a rabbit anti-Ngb poly- clonal antibody (Bs-1858r, Beijing, China) for one night at $4^{\circ} \mathrm{C}$. The sections were rinsed in PBS and incubated at room temperature for 80 minutes. The sections were re-washed and embedded in Trypsin (T-815025, Beijing, China). After that, the sections (70\% alcohol (night), $80 \%$ alcohol (5 h), $90 \%$ alcohol (3 h), 95\% alcohol ( $2 \mathrm{~h}$ ), and n-butanol (night) converted to $\mathrm{n}$-butanol, $(5 \mathrm{~h})$ were prepared and embedded into paraffin wax, tissue sections were cut and examined under an optical microscope (Olympus BX51).

Reagents and instruments. The Trypsin (T-815025), Phosphate-buffered saline (PBS, Zli-9062) and rabbit anti-rat polyclonal antibody (Bs-1858r) were purchased from Beijing Bio-Dee Biotechnology Co. Ltd. while the immunohistochemical staining kit (sp-9001) and DAB color developing kit (Zli-9018) were ordered at Beijing Zhongshan Jinqiao Biotechnology Co. Ltd and Formaldehyde and n-butanol solution, analytically pure came from Tianjin Baishi Chemical Co. LTD. Glacial acetic acid and alcohol solution: analysis-pure were bought from Tianjin Fuyu Fine Chemical Co Ltd and the normal sheep serum (ab7489) came from North America. The following medical instruments were purchased and employed during the sampling procedures: conventional surgical instruments which include: scalpel and dissecting scissors, biological tissue embedding KD-BM machine, Bio-texturing and spreading machine (K-P), and Biological tissue baking machine (KD-H) were purchased from Zhejiang Medical Getidy Medical Instruments Co. Zhejiang, Jinhua. The Constant temperature incubator DHP-9082 was ordered at Shanghai Tajilin Industrial Co. Ltd, while the Semi-self-actuating screw-cutting machine with a precision roller (Rm-2235) was bought from Leica, Germany, and Optical microscope $(13395 \mathrm{H} 2 \mathrm{X})$ was purchased at Leica, Germany.

Immuno-histochemical coloration. Streptavidin Peroxidase (SP) method was used for immunohistochemical staining while PBS was utilized as a negative control instead of the primary antibody. Sheep serum was added to block the non-specific reactivity in each section and then observed under the optical microscope (Olympus 13395H2X) to control color rendering. The color rendering period for each section was consistent. After the reaction stopped by distilled water, the slices were re-dyed, dehydrated, and transparent by hematoxylin and sealed. A Nikon Eclipse 80i, micro camera system was employed to capture specimen images with consent shooting parameters and the ImagePro Plus 6.0 was introduced to acquire, count, measure, and classify optical density of each retina layer.

Statistical analysis. Data analysis was performed using Statistical Package Social Sciences (SPSS) 1.0 (1983) and 19.0 (2009) to conduct one-way ANOVA, multiple comparisons (Tamhane, ST2 method), and the Independent Sample T-test was employed to evaluate the significant level. $\mathrm{P}<0.05$ was considered significant. If there existed no significant difference in the $\mathrm{MD}$, it was referred to as the negative control $(\mathrm{P}>0.05)$, and positive $(+)$ control or expression if there was any significant difference.

\section{Results and discussion}

Adult Bactrian camel. The reports from HE staining showed that the Bactrian camel structure contains 

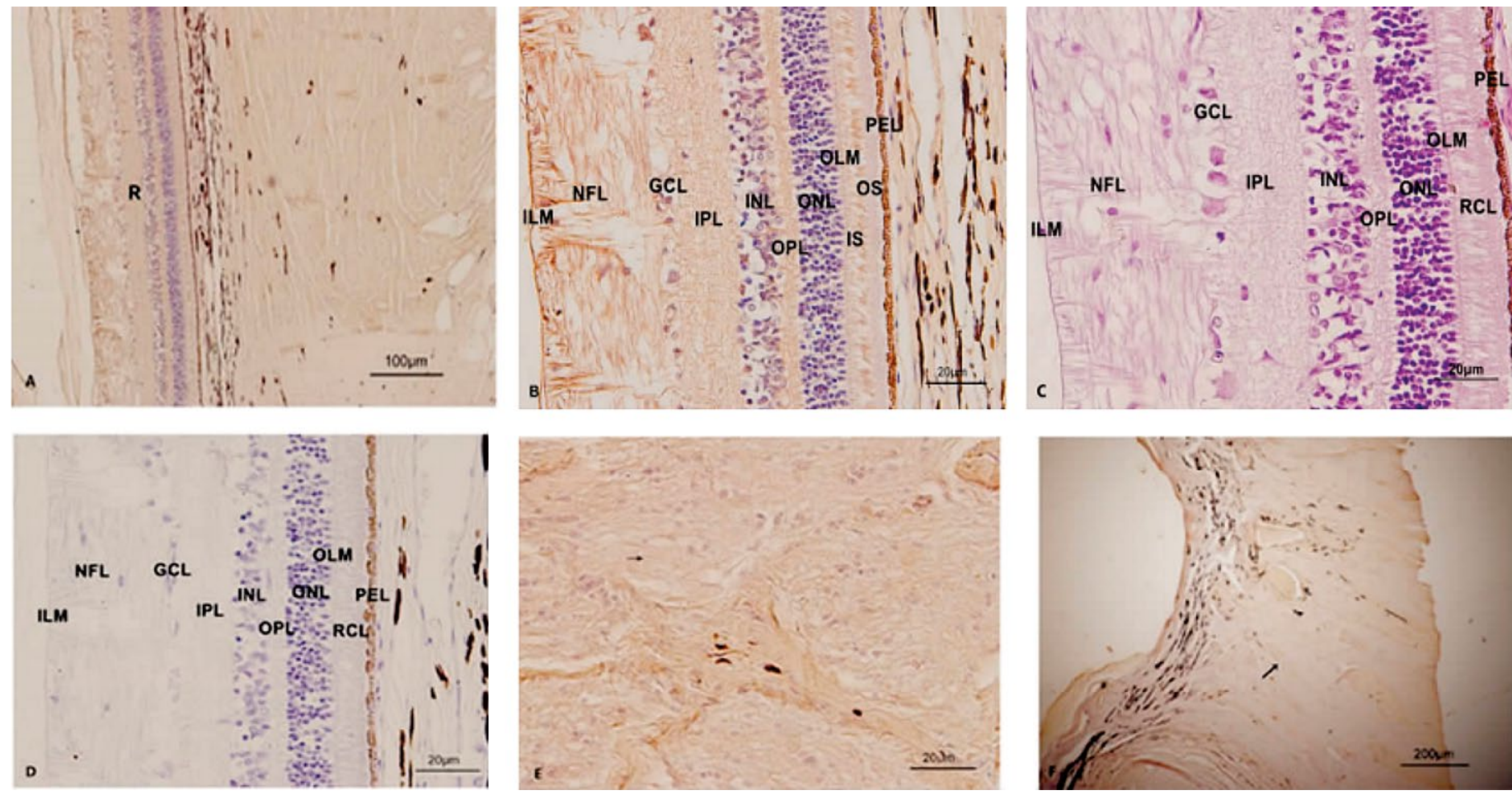

Fig. 1. Distribution of neuroglobin in the retina of the adult Bactrian camel

10 layers with normal cell stratification, which are similar to other mammals. The characteristics of each layer are described as follows: The inner boundary membrane contains the miller and glial cells, accompanied by vitreous fibers covering the whole retinal surface. It is referred to as the innermost layer of the homogeneous film. While the optic nerve fiber layer, when examined under the light microscope, is a crisscrossed and porous network structure which composed of ganglion cell axons and has small blood vessels that were observed. The ganglion cell layer is composed of clearly arranged ganglion cells and nucleus of different sizes and the inner reticular layer: a dense reticular structure formed by the protuberance of nodal cells and bipolar cells; on the other hand, the core layer is described as the loose nuclear layer composed of bipolar and horizontal cells which can be observed under the light microscope. The outer reticular layer is a dense reticular structure composed of dendrites of horizontal and bipolar cells and axons of photoreceptor cells and the outer nuclear layer contains a dense nuclear layer composed of photoreceptor cells with a tight arrangement and clear nucleus which can be examined under the light microscope. The outer membrane is a thin and clear membrane structure composed of muller cells and the rod-cone layer is a region composed of rod and cone cells, which can be divided into two parts under the light microscope: internal and external segments of photoreceptors. Another region is the pigment super cortex which is located in the outermost part of the retina and consists of pigment cells and flat cells formcamel ing a dark cell layer (Fig. 1C). The layers of cells form a complex neural network in the horizontal and vertical directions to complete the processing of information. The immuno-histochemical results showed that Ngb expression was detected in all layers except the outer nuclear layer of Bactrian camel retina (Fig. 1A and 1B). At the cellular level, Ngb is expressed intensively in the cytoplasm of pigment epithelial cells, photoreceptor cells, bipolar cells, and nodal cells. No positive expression was found in the negative control group (Fig. 1D). Moreover, positive $\mathrm{Ngb}$ distribution was observed in the optic nerve of the Bactrian camel (Fig. 1E and 1F). The reported results of Intel's integrated performance primitives (IPP) and statistical analysis showed that the MD value of the outer nuclear layer was 0.0334 \pm 0.0144 , explaining no significant existence in the negative $(-)$ control group $(\mathrm{P}>0.05)$. The $\mathrm{MD}$ values

Tab. 1. The comparison of the Ngb expression intensity in adult Bactrian

\begin{tabular}{|l|c|c|}
\hline \multicolumn{1}{|c|}{ Regions } & Main Optical Density & The Expression Density \\
\hline Nerve fiber layer (NFL) & $0.1762 \pm 0.0707^{* * \Delta}$ & ++ \\
Ganglion cell layer (GCL) & $0.1611 \pm 0.0491^{* \star \Delta}$ & ++ \\
\hline Inner plexiform layer (IPL) & $0.1424 \pm 0.0464^{* * \Delta}$ & ++ \\
\hline Inner nuclear layer (INL) & $0.0794 \pm 0.0238^{* * \Delta}$ & + \\
\hline Outer plexiform layer (OPL) & $0.1407 \pm 0.0453^{* * \Delta}$ & ++ \\
\hline Outer nuclear layer (ONL) & $0.0334 \pm 0.0144^{*}$ & - \\
\hline Photoreceptor inner segments (IS) & $0.1756 \pm 0.0502^{* * \Delta}$ & ++ \\
\hline Photoreceptor outer segments (OS) & $0.0749 \pm 0.0229^{*}$ & + \\
\hline Pigmented epithelial layer (PEL) & $0.1985 \pm 0.0803^{* * \Delta}$ & ++ \\
\hline
\end{tabular}

Explanations: * positive and negative control group, $\mathrm{P}>0.05 ; * *$ positive and negative control group, $\mathrm{P}<0.05 ; \stackrel{\wedge}{ }$ positive control group, $\mathrm{P}<0.05$ 
of each region were significantly different from the negative contrast $(\mathrm{P}<0.05)$, and were determined to be a weakly positive $(+)$ expression. Similarly, the regions with MD values were higher than the kernel layer, and the significant difference $(\mathrm{P}<0.05)$ was considered as a strongly positive $(++)$ expression, which was consistent with the observation results under the optical microscope (Tab. 1).

Adult rabbits. In the retina of adult rabbits, the $\mathrm{Ngb}$ immuno-positive reaction was mainly distributed in the optic nerve fiber layer, ganglion cell layer, inner reticular layer, outer reticular layer, the inner segment of photoreceptor and pigment super-cortex, inner boundary layer, outer membrane and inner layer (Fig. 2). The Ngb positive expression found in the fibril was mainly located in the axon of ganglion cells while a similar expression in the nodal cell layer was observed in the body of nodal cells with different sizes and specific lining. Immuno-histochemical results showed that $\mathrm{Ngb}$ was localized in the cytoplasm, and no positive staining was observed in the nucleus. Ngb positive expression in the inner reticular layer was mainly the axons of bipolar cells and the dendrites of ganglion cells. The expression of $\mathrm{Ngb}$ in the outer reticular layer is the axons of rods and cones, the dendrites of bipolar cells, and the protuberances of horizontal cells. The pigment epithelial layer contains a positive expression of $\mathrm{Ngb}$ and it is located in the pigment epithelial cells. The positive expression of the inner layer was the cell body of bipolar cells, in which the cytoplasm was strongly colored. The cytotypes of $\mathrm{Ngb}$ positive phenotype are mainly photoreceptor cells, bipolar cells, and retinal ganglion cells, which are consistent with the third-order retinal neurons. No Ngb positive expression was found in the outer nuclear layer and the outer segment of the photoreceptor in the retina of adult rabbits. There were no positive signs in the negative control group (Fig. 2 v). Besides, immunization results showed that positive expression of $\mathrm{Ngb}$ was observed in the optic nerve of adult rabbits (Fig. 2 iii, iv) and the statistical analysis are shown in Tab. 2.

Adult sheep. The retina is the innermost layer of the mammalian eye which contains ten layers from inner to the outer one (Fig. 3 $\mathrm{i}$-vi). In the retina of adult sheep, the $\mathrm{Ngb}$ was mainly distributed in the optic nerve fiber layer, ganglion cell layer, inner and outer reticular layer, the inner segment of the photoreceptor, while the positive expression was found in the inner and outer membranes (Fig. 3). The positive expression of the optic nerve fiber layer was mainly located in the axon of ganglion cells and the positive surface of the nodule cell layer was considered as the cell body of the nodule cell with different sizes and outlines. Immunohistochemical results showed that Ngb expression was located in the cytoplasm, and no positive staining was observed in the nucleus. The Ngb positive expression of the inner reticular layer was distributed in the axon of the bipolar cell and dendrite of the node cell, while in the outer reticular layer it was found in the axons of rods and cones, the dendrites of bipolar cells and the protuberances of horizontal cells. The positive expression of $\mathrm{Ngb}$ in the inner layer was the cell body of bipolar cells, in which the cytoplasm was strongly

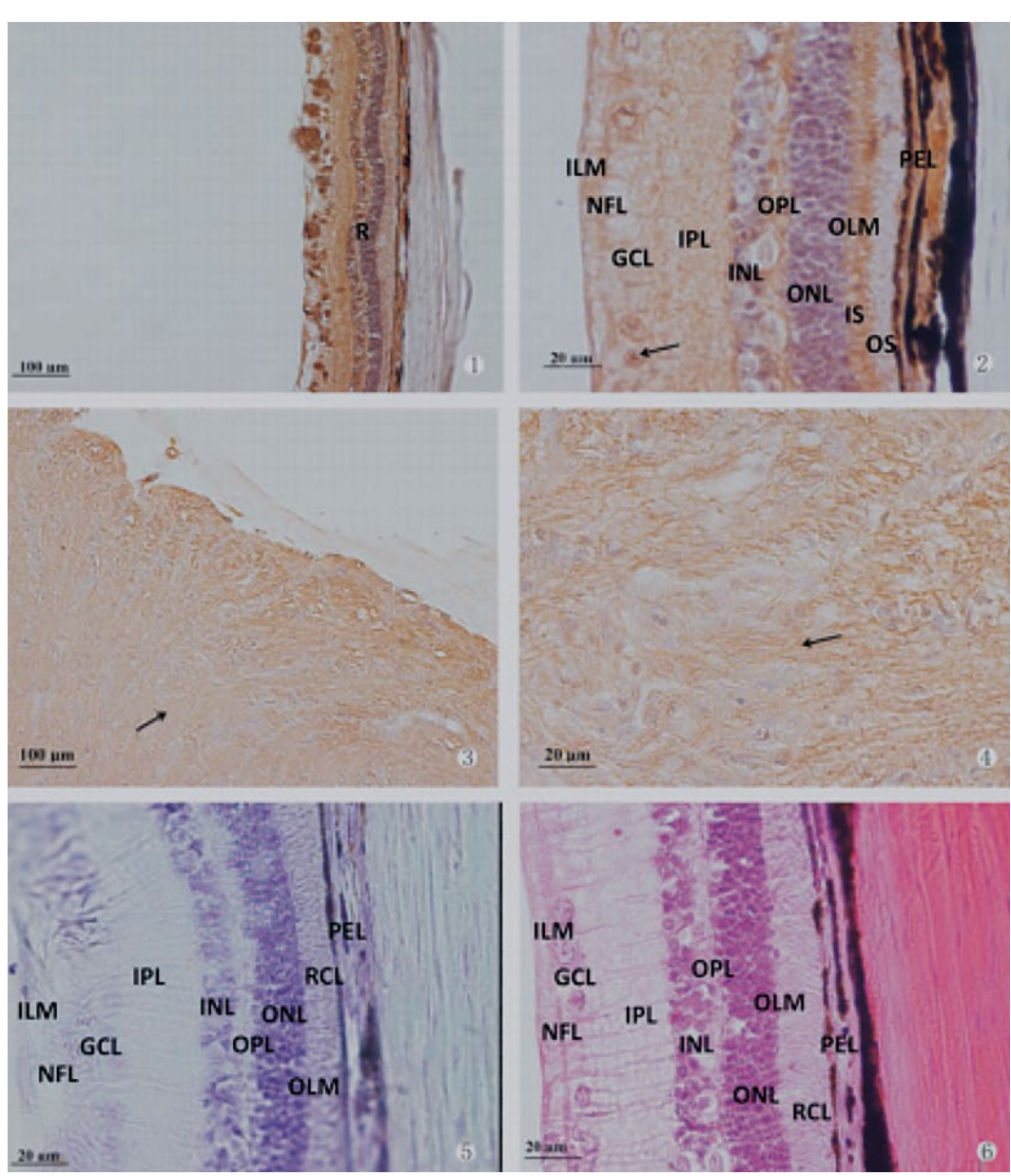

Fig. 2. Distribution of neuroglobin in the retina of the adult rabbit

Tab. 2. The comparison of the Ngb expression intensity in the adult rabbit

\begin{tabular}{|l|c|c|}
\hline \multicolumn{1}{|c|}{ Regions } & Main Optical Density & The Expression Density \\
\hline Nerve fiber layer (NFL) & $0.151 \pm 0.050^{* * \Delta}$ & ++ \\
\hline Ganglion cell layer (GCL) & $0.173 \pm 0.040^{* * \Delta}$ & ++ \\
\hline Inner plexiform layer (IPL) & $0.140 \pm 0.019 * * \Delta$ & ++ \\
Inner nuclear layer (INL) & $0.091 \pm 0.031^{* *}$ & + \\
\hline Outer plexiform layer (OPL) & $0.123 \pm 0.017^{* * \Delta}$ & + \\
\hline Outer nuclear layer (ONL) & $0.020 \pm 0.013^{*}$ & + \\
\hline Photoreceptor inner segments (IS) & $0.143 \pm 0.038^{* * \Delta}$ & + \\
\hline Photoreceptor outer segments (OS) & $0.033 \pm 0.014^{*}$ & ++ \\
\hline Pigmented epithelial layer (PEL) & $0.170 \pm 0.040^{* * \Delta}$ & + \\
\hline
\end{tabular}

Explanations: as in Tab. 1. 

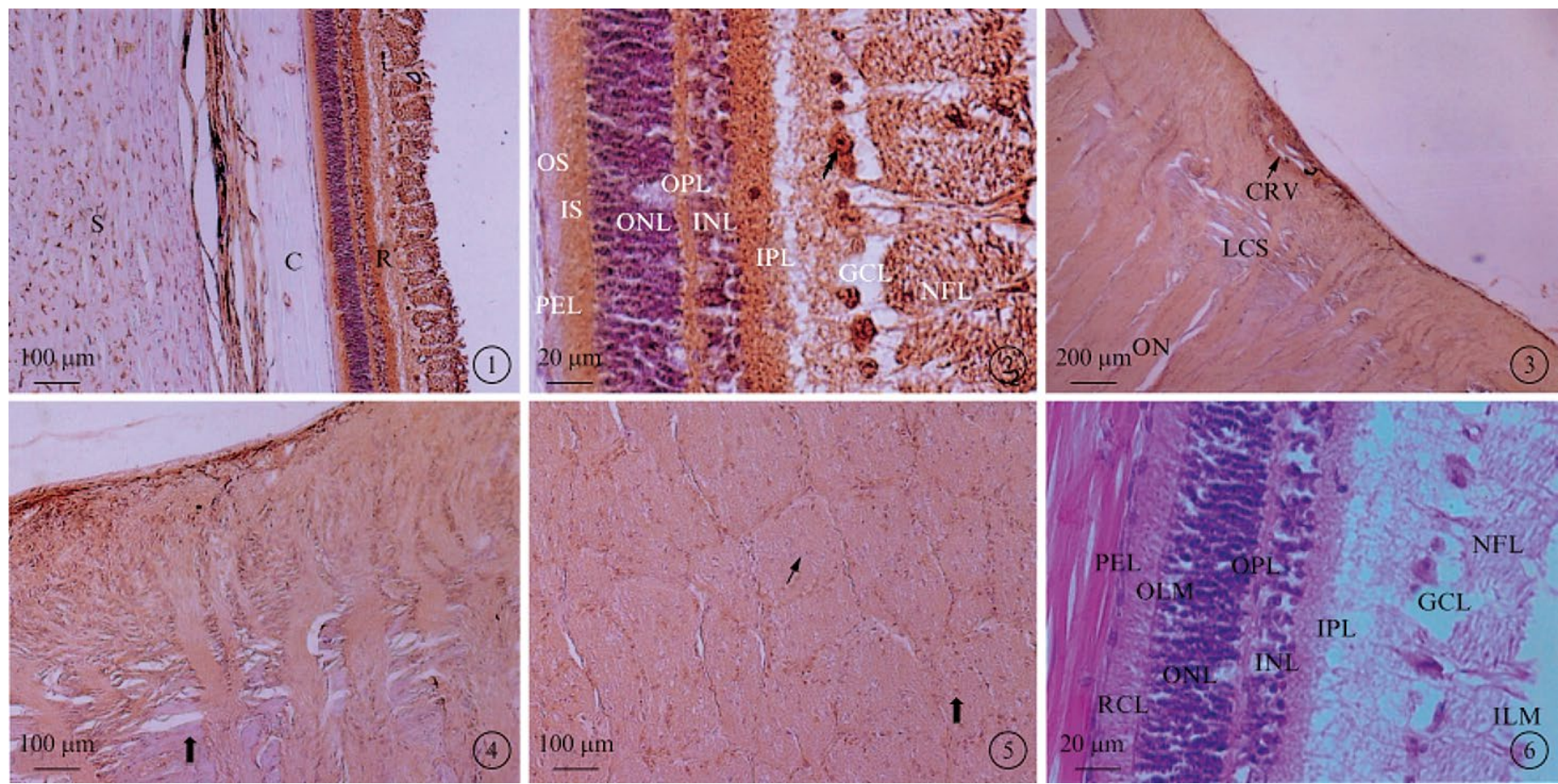

Fig. 3. Distribution of neuroglobin in the retina of adult sheep

colored. The main positive expression of cell types was the photoreceptor cells, bipolar cells, and retinal ganglion cells, which were consistent with the third-order neurons in the optic network membrane. No expression of $\mathrm{Ngb}$ exists in the outer nuclear layer of adult sheep retina, the outer segment of the photoreceptor, and the upper layer of chromatin. The Image-Pro Plus 6.0 semi-quantitative analysis software reported that the positive control group of sheep retinal optic nerve fiber layer, thin cell layer, inner plexiform layer, kernel layer, outer plexiform layer, the outer nuclear layer, photoreceptor inner segment, and pigments on the cortex photoreceptor outer segments of Median Derivative (MD) values are mentioned (Tab. 3). The T-test results read that the outer nuclear layer of the positive control group and pigments on the cortex photoreceptor outer segments showed no statistical significance as compared with the negative $(-)$ control group $(\mathrm{P}>0.0)$. The positive control group MD value of the optic nerve fiber layer, inner plexiform layer, network layer, and photoreceptor inner segment were higher than the ganglion cell layer and kernel layer $(\mathrm{P}<0.05)$, indicating that the optic nerve fiber layer and other layers were strongly positive $(++)$. The ganglion and kernel cell layers were weakly positive $(+)$, which was consistent with the visual observation of positive expression under a light microscope. Ngb was also positively expressed in the optic nerve of sheep (Fig. 3i). Immunohistochemical results showed that except for the positive expression in the sclera sieve plate, positive expression of Ngb was found in nerve fibers, glial cells, and central vascular wall of the retina (Fig. 3 iv, v).
Tab. 3. The comparison of the Ngb expression intensity in sheep

\begin{tabular}{|l|c|c|}
\multicolumn{1}{|c|}{ Regions } & Main Optical Density & The Expression Density \\
\hline Nerve fiber layer (NFL) & $0.152 \pm 0.011^{* * \Delta}$ & ++ \\
Ganglion cell layer (GCL) & $0.110 \pm 0.007^{* * \Delta}$ & ++ \\
\hline Inner plexiform layer (IPL) & $0.121 \pm 0.011^{* * \Delta}$ & ++ \\
\hline Inner nuclear layer (INL) & $0.078 \pm 0.208^{\Delta}$ & - \\
\hline Outer plexiform layer (OPL) & $0.015 \pm 0.001^{* * \Delta}$ & ++ \\
\hline Outer nuclear layer (ONL) & $0.013 \pm 0.001^{* *}$ & ++ \\
Photoreceptor inner segments (IS) & $0.085 \pm 0.012^{* * \Delta}$ & ++ \\
\hline Photoreceptor outer segments (OS) & $0.016 \pm 0.004^{* *}$ & ++ \\
\hline Pigmented epithelial layer (PEL) & $0.018 \pm 0.012^{* * \Delta}$ & ++ \\
\hline
\end{tabular}

Explanations: as in Tab. 1.

Adult Bactrian. The reported results in the current study showed that $\mathrm{Ngb}$ is positively expressed in all layers of the Bactrian camel retina except the outer retinal nucleus, which is consistent with the results of previous studies on the adult rat, sheep and human (2, $9,16,17)$. Different research viewpoints are held on the distribution of $\mathrm{Ngb}$ in the upper chromatin cortex. Bentmann et al. (3) found that there was no Ngb positive substance in the pigment super cortex of rats and mice. However, Ostojic et al. (18) found that there was Ngb positive expression in the pigment super cortex of humans and dogs. Yang et al. (28) studies reported that there was Ngb distribution observed in the pigment super cortex of rabbits, but results on the sheep did not find Ngb expression in the super cortex pigment. Similarly to these reported results, the current study also found $\mathrm{Ngb}$ positive distribution in the retinal pigment of the Bactrian camel's super cortex. These differences between previous results may be caused by different animal species, different living 
environments, different specificity of antibody quality employed, and immunological detection. These changes lead to the different expression and localization of neuroglobin which are suggested to influence the adaptive potential of an organism (1). The pigment cortex is located in the outermost layer of the retina, and its main function is to supply oxygen and nutrients to the outer retina and absorb light scattering. Therefore, the pigment super cortex is vulnerable to the photochemical damage of visible light, which leads to the accumulation of reactive oxygen species (ROS), and then produces oxidative stress reaction, resulting in cell apoptosis. However, Ngb can remove excessive ROS, maintain the oxygen balance of tissue cells, and protect cells from light damage, which may also be one of the reasons for the different Ngb expression. Other results confirmed that oxygen affinity and enhanced auto-oxidation question the role in oxygen delivery and proposed that the neuroprotective effect might be due to radical scavenging or activation of protection mechanisms (4).

Researchers are still debating the exact location of $\mathrm{Ngb}$. Ran et al. (19) found that there were strong $\mathrm{Ngb}$ mRNA hybridization signals in the inner and outer nuclear layers of rats. However, the study of Xu et al. (24) showed that Ngb was not expressed in the outer nuclear layer. The results of this current study show that $\mathrm{Ngb}$ is not distributed in the outer nuclear layer, which is consistent with Xu's report. Some studies documented that there might be certain differences in the spatial distribution of Ngb mRNA and protein in the retina, which makes the retinal structure of mammals to be partitioned. Ngb mRNA is mainly distributed in the cell body, while Ngb is distributed in the protrusion, suggesting that the Ngb protein has a transport process after translation. In the present research, the positive distribution of $\mathrm{Ngb}$ in the outer segment of the photoreceptor is rarely reported. The current results revealed the presence of $\mathrm{Ngb}$ in the outer segment of the photoreceptor. This result may be related to the different animal species or may be closely related to the function of the outer segment of the photoreceptor during the visual formation of the Bactrian camel under extreme environmental conditions. Whether $\mathrm{Ngb}$ is related to the adaptability of the environment remains to be studied. The Bactrian camel lives in an extreme natural environment for a long time, with a dry climate and strong ultraviolet rays. A long period of illumination leads to excessive production of ROS and synthesis of lipid peroxidation product MDA, resulting in retinal damage. Such lesions are mainly located in the regions of mitochondria in the retina (20), because the inner segment of the photoreceptor is rich in mitochondria, while the outer segment is rich in polyvalent unsaturated fatty acids and prone to the formation of MDA, which may cause the rod and cone layer to be damaged by ROS and MDA. Fordel et al. (12) studied sh-sy5y nerve cells and found that when Ngb protein expression increased or decreased, it was accompanied by a corresponding decrease or increase in $\mathrm{H}_{2} \mathrm{O}_{2}$ content, indicating that $\mathrm{Ngb}$ was a ROS scavenger. On the other hand, Nayak et al. (16) found that $\mathrm{Ngb}$ produced by cultured nerve cells in vitro can reduce ROS levels, indicating that Ngb plays a certain role in ROS elimination. Similarly, the current results have shown that Ngb positive expression of different intensity was found in the rods and cones and the super cortex pigment, indicating that Ngb may have an important repairing effect on retinal damage caused by light. However, further research is needed to confirm this report. The main function of the retina is visual conduction in which photoreceptors convert the received photochemical signals into electrical signals, and can be transmitted to the central nervous system through various neurons in the optic network membrane forming vision. The current study reported that Ngb was expressed in the cytoplasm, rods, cones, and other cells of the retinal microcytes and bipolar cells. However, other studies have shown (22) that there are a large number of synaptic connections of adjacent binary neurons between ganglion cells, bipolar cells and rod-cone cells to transmit information, which is one of the regions with the highest oxygen consumption in the retina and also the region with concentrated mitochondria, so it is very sensitive to oxidative damage caused by ROS. The high expression of $\mathrm{Ngb}$ as a ROS scavenger in mitochondria suggests that $\mathrm{Ngb}$ is maintaining a high oxygen consumption group that has more complex physiological functions in tissue metabolism. $\mathrm{Ngb}$ is expressed with different intensity in each layer of the retina, and its expression level is positively correlated with the demand for tissue cells for oxygen. Moreover, Ngb is an endogenous neuroprotective agent, and the expression level significantly increased due to hypoxia and ischemia of tissue cells (21). Roberts et al. (20) who employed the method of constructing a mathematical model showed that the effect of $\mathrm{Ngb}$ is not in the oxygen storage, but the oxygen may play a more important role in the models to predict photoreceptors in the retina anoxic area in the segment and inner plexiform layer which is involved in the functioning of high expression of $\mathrm{Ngb}$ area. The expression of Ngb strength with the regional demand for oxygen utilization is closely related. Moreover, the distribution of $\mathrm{Ngb}$ was also found in the optic nerve of the Bactrian camel, providing clues for further research on the physiological function of $\mathrm{Ngb}$ in protecting the optic nerve under extreme living conditions. The histological study on the expression distribution of $\mathrm{Ngb}$ in the retina of the Bactrian camel showed that the expression distribution of $\mathrm{Ngb}$ in the retina of the Bactrian camel was similar to that of other mammals, but also had its own characteristics. The positive expression of $\mathrm{Ngb}$ in the outer segment of the photoreceptor of the Bactrian camel's retina enriches the morphological information of the distribution of 
$\mathrm{Ngb}$ in the mammalian retina and provides valuable clues for the study of its function.

Adult rabbits. Previous studies have shown that $\mathrm{Ngb}$ was positively expressed in all layers of the rodent, dog, and human retinas, excluding the pigmented cortex and photoreceptors $(7,23)$. The studies further stated that $\mathrm{Ngb}$ is highly concentrated in the layers and expression levels differ. Similarly, the current results reported that Ngb expression was found in these regions. The positive distribution of $\mathrm{Ngb}$ was observed in the outer reticular layer, outer membrane and the inner segment of the photoreceptor, while no positive expression was observed in the outer segment of the photoreceptor, retinal inner boundary membrane, optic nerve fiber layer, ganglion cell layer, inner reticular layer, and kernel layer. Up to the present, debate on the exact localization and mechanism of $\mathrm{Ngb}$ remains a focus among scientists. A study documented that $\mathrm{Ngb}$ show sequence variation in vertebrate evolution, suggesting conserved structures and functions, and an important role in the animal's metabolism (5). Li You (15) et al. utilized immunohistochemistry to confirm $\mathrm{Ngb}$ expression, and the results reported that the expression of $\mathrm{Ngb}$ was observed in other layers except for the pigmentation cortex of rats and mice. However, Ostoji et al. (17) applying similar methods found that $\mathrm{Ngb}$ was located in the retinae of humans. The expression was mainly located in the upper epidermis of human and dog retinas. In the current study, Ngb positive expression was observed in the superior cortex of the retinal pigment in adult rabbits which is consistent with the observation of Ding et al. (11). There also exists a minimum difference in these results and the difference may have originated from the sensitivity of the antibody and the methodology employed. Additionally, to ensure the functionality of the retina, humans and most mammals through the retina and choroid double loop to oxygen in a row, the inner retinal nutrition by the central retinal vascular system, the outer retinal blood supply is mainly supported by choroid cycle, and the circulation of choroid blood does not directly reach the photoreceptor cell layer, which must pass through a pigment in the cortex of oxygen to diffuse to the photoreceptors. This might be one of the reasons for different $\mathrm{Ngb}$ expression. Whether the presence of Ngb in the pigment super cortex is produced by the pigment super cortex cells or the transport and redistribution of $\mathrm{Ngb}$ produced in neurons remains to be verified by further experiments. As reported in the study of Ding et al. (10). Ngb is distributed in the mammalian retina outer nuclear layer which differs from the current research report. The in situ hybridization histochemistry technique was used by Yan et al. (27) and Ostoji (17) to examine the expression of $\mathrm{Ngb}$ in the inner and outer nuclear layer in the retinae of human and rat. The results confirmed that strong expression was found in the inner and outer nuclear layers which are similar to what is re- ported in the current study but differs from the results of Xu et al. (25). The difference may be related to the sensitivity of the antibodies and methods used. Immuno-histochemical semi-quantitative analysis results showed that $\mathrm{Ngb}$ expression in rabbit retina layers has an obvious difference on the strength of the section in the optic nerve fiber layer, cell layer and inner plexiform layer, outer plexiform layer and optical sensors in segmental positive expression which has the highest intensity. This is consistent with what Schmidt et al. reports in the retina of mice (21). Studies have shown that the optic nerve fiber layer, ganglion cell layer, inner reticular layer, outer reticular layer and inner photoreceptor segment of the mammalian retina are the most concentrated parts of the retina in oxygen consumption because these regions are the adjacent layer of the synaptic connection between neurons and mitochondria (27). These neurons are involved in the passage of information through mutual synapses. The photoelectric signal conversion needs to consume large amounts of oxygen, and Ngb in these regions is highly expressed. These regions and the subcellular localization of mitochondria are closely related since with the expression of $\mathrm{Ngb}$ because the body carrying oxygen can also be used by retinal neurons to satisfy the demand of oxygen consumption. Additionally, Xu et al. (25) found that there were more Ngb positive particles distributed between the synaptic vesicles in the presynaptic structure of the adult rat retina, which supported the hypothesis that $\mathrm{Ngb}$ was related to the oxygen consumption of the optic network membrane. This further suggested that Ngb could be secreted and transported to the synaptic vesicles to complete complex physiological functions. The present study likewise found that $\mathrm{Ngb}$ also had a high level of positive expression in the retinal pigment superior cortex of rabbits. Studies have shown that the inner folds of the plasma membrane of the pigment epithelial cells can expand the contact area between the pigment epithelial cells and the choroidal capillaries, which is conducive to the selective transfer of nutrients and metabolites between the outer layer of the retina and the choroidal capillaries (29). Moreover, epigallocytes have the functions of vitamin A transport and metabolism, drug detoxification, synthesis of melanin and extracellular matrix, and phagocytosis and digestion of membrane plates shed from the outer segments of photoreceptors. These functions of pigment epithelial cells all require higher energy requirements on themselves, suggesting that the high expression of Ngb in the pigment epithelial layer may be related to the high oxygen consumption needed to maintain normal functional activities. Studies have shown that Ngb plays a crucial role in the dynamic oxygen balance of the retina, and its existence can make the optic network membrane endure longer under the condition of ischemia and hypoxia (20). Shi et al. (22) found that Ngb was rapidly increased after the lack of blood in the 
optic network membrane, indicating that $\mathrm{Ngb}$ is very sensitive to hypoxia, suggesting that $\mathrm{Ngb}$ may play an important role in the adaptive protection of retinal hypoxia, while Ding et al. (11) showed that in the acute ischemic and hypoxic state the main concentration of $\mathrm{Ngb}$ content in the retina of rats was the ganglion cell layer, and a small amount of $\mathrm{Ngb}$ content was also found in the inner and outer netted layer. In addition, Yan et al. (27) explained that the expression of Ngb in the retinal tissues of rabbits was higher than that in the plain and subalpine environments under the hypoxic environment, while $\mathrm{Ngb}$ in the plain area was only weakly expressed in the inner and outer plexus layers and the inner segment of photoreceptors. The present research results show that the expression of $\mathrm{Ngb}$ in the optic nerve fiber layer and ganglion cell layer and inner plexiform layer, outer plexiform layer, photoreceptor inner segment and the intensity of expression of the highest in areas such as the pigment in the cortex, expressed in the kernel layer strength is weak, prompt retinal optic nerve fiber layer and ganglion cell layer and inner plexiform layer, outer plexiform layer and optical sensors in segmental oxygen consumption, such as the concentration of the highest place on the degree of sensitivity of ischemia hypoxia, cortex cell metabolism active pigment, with special phagocytosis. At the same time, it also performs the function of biofiltration of the sensory layer of the retina, which requires a higher energy supply, so it is sensitive to ischemia and hypoxia the highest. Additionally, the inner core layer acts as a pathway for transmitting photoreceptor cell impulses to ganglion cells, both of which have large oxygen consumption requirements. The sensitivity of ischemia and hypoxia was also higher, while the areas with negative Ngb expression, such as the outer nuclear layer and the outer segment of the photoreceptor, were the least sensitive to hypoxia. Therefore, the difference of Ngb expression in each layer of the retina may also be correlated with the sensitivity of each layer to ischemia and hypoxia injury.

Adult sheep. Existing research has shown that Ngb is solely expressed in rodent, dog, and human retinae excluding the pigment in the cortex and light sensor section which contain no expression of $\mathrm{Ngb}$. A study conducted on the mouse retina found that Ngb is expressed in all neurons especially the plexiform layers and the ellipsoid region of the photoreceptor inner segment (7), which is consistent with the current research that showed expression in the plexiform layers. Ostojic et al. studied the retina in canines (dog) and reported that $\mathrm{Ngb}$ is solely expressed in the ganglion cell layer, inner and outer nuclear layers, inner and outer plexiform layers, photoreceptor inner segments, and retinal pigment epithelium. By using immuno-histochemical Schmidt (21) and $\mathrm{Li}(15)$ confirmed that Ngb is not expressed in the layer of the skin color but can be found in other layers of the small rat retina, but by applying a similar method Ostojic I $(17,18)$ showed that $\mathrm{Ngb}$ is observed in all retina layers, especially the cortex of retina pigment in the human and dog. Despite these reported references, there are still limited reports on the retina of adult Tibetan sheep. The results of this study showed that there were positive distributions of $\mathrm{Ngb}$ in the inner boundary membrane, optic nerve fiber layer, ganglion cell layer, inner reticular layer, inner layer, outer reticular layer, outer membrane and an inner segment of the photoreceptor of the adult sheep retina but no positive expression was found in the outer segment of the photoreceptor, pigment super cortex, and optic network membrane which is consistent with the findings of Bentmann et al. (2), but different from the observation results of Ostojic et al. (17). The expression in these regions has shown to be strongly expressed with white mRNA of mixed-signal. Yan et al. and Ostojic et al. $(18,27)$ also applied the immunohistochemistry method to obtain the expression of $\mathrm{Ngb}$ and immune response in the retina of rats and humans. The Ngb distribution in both rats and humans was consistent but in their study Xu et al. (24) did not observe any Ngb distribution in the outer nuclear layer sub-cells of the adult rat retina. Similarly, the current reports also observed no positive $\mathrm{Ngb}$ expression in the outer nuclear layer of adult sheep retina. To ensure the network of membrane function, human and most mammalian retina and choroid membrane double loop to continue the flow of oxygen. Central retinal nutrition blood pipe system and outer retinal blood supply are mainly supported by the choroid cycle, and the circulation of choroid blood does not directly reach the photoreceptor cells layer, which must pass through a pigment in the cortex of oxygen to diffuse to the light receptors. The results of the immuno-histochemical semi-quantitative analysis showed that there were significant differences in the expression of $\mathrm{Ngb}$ in different layers of the sheep retina. The positive expression intensity was recorded as the highest in the dimension layer, the inner reticular layer, the outer reticular layer, and the inner segment of the photoreceptor, which was similar to the study results of Schmidt et al. (21) who studied Ngb expression in the retinae of mice. Mammalian retinal nerve fiber layer, inner plexiform layer, outer plexiform layer, and optical sensors are the most important parts of oxygen consumption in retinal tissue $(2,3)$, due to the great amount of synaptic coupling between adjacent layer neurons and mitochondria. The neurons send information through mutual synapses and releasing the photoelectric signal conversion. This process requires a large amount of oxygen consumption and the expression of $\mathrm{Ngb}$ was showed to be higher in these regions which are related to the subcellular localization of mitochondria. The oxygen transported can be utilized by retinal neurons to satisfy the high oxygen demand of the four layers. In the subcellular structure, the $\mathrm{Ngb}$ protein is not found in the mitochondria but around them, which has important implications for further 
exploration of the physiological mechanism. It also enhances cell viability under hypoxia and under various types of oxidative stress in transgenic systems, but does not appear to be strongly upregulated in response to stress (6). Additionally, Xu et al. (25) reported that the $\mathrm{Ngb}$ distribution in the presynaptic structure of rat retina between synaptic vesicles have more levels of $\mathrm{Ngb}$ expression in males than females and further supports the Ngb protein which is assumed to be associated with the retinal oxygen consumption. This has suggested that $\mathrm{Ngb}$ protein can be secreted by the neurons and transported between synaptic vesicles to complete complex physiological functions. The expression of Ngb in different retinal layers may correspond to the sensitivity of each layer to ischemic and hypoxic injury. Ding et al. (10) found that in the acute ischemic and hypoxic state, the increase of Ngb in the cerebral of the rat's retina was mainly concentrated in the nodule cell layer, and less expression observed in the inner and outer reticular layer. The experimental results of the current study also reported that Ngb showed high intensity in the optic nerve fiber layer, network layer, outer plexiform layer, and the light receptors. In areas such as the ganglion cells and kernel layer, the strength of $\mathrm{Ngb}$ expression is weak. Other layers including the optic nerve fiber layer, inner and outer plexiform layer, and optical sensors consumed the highest level of oxygen and they are the most concentrated areas of high ischemia hypoxia sensitivity. In addition, the thin cell layer channels information to the brain while the retina kernel layer as impulse transfer photoreceptor cells to access the ganglion cells. Both have a high demand for oxygen, and are highly sensitive to ischemia hypoxia. The outer nuclear layer, photoreceptor outer segments, and pigment on the cortex of the brain showed Negative Ngb expression and express least sensitive to the demand for oxygen.

The reported results have suggested that Ngb may have an important physiological role in maintaining retinal oxygen homeostasis and participating in the repair mechanism of light damage in extreme survival to adapt to environmental conditions. The study further provides a theoretical basis for in-depth research of the $\mathrm{Ngb}$ physiological function in the retina and recorded morphological data for further exploring the distribution of $\mathrm{Ngb}$ in normal mammalian retinas. However, the mechanism of $\mathrm{Ngb}$ involvement in damage repair after retinal injury remains to be studied further in molecular and tissue biology.

\section{References}

1. Alekseeva O. S., Grigorev J. P., Korzhewskij D. E.: Neuroglobin, an oxygenbinding protein in the mammalian nervous system (localization and putative functions). J. Evolut. Biochem. Physiolog. 2017, 53, 249-258.

2. Anderson $B$.: Ocular effects of changes in oxygen and carbon dioxide tension. Trans Am. Ophthalmol. Soc. 1968, 66, 423-474.

3. Bentmann A., Schmidt M., Reuss S., Wolfrum U., Hankeln T., Brumester T.: Divergent distribution in vascular and avascular mammalian retinae links neuroglobin into cellular respiration. J. Biochem. 2005, 280, 21, 20660-20665.
4. Brunori M., Vallone B.: Neuroglobin, seven years later. Cell Mol. Life Sci. 2007, 64, 1259-1268, doi: 10.1007/s00018-007-7090-2.

5. Burmester T., Haberkamp M., Mitz S., Roesner A., Schmidt M., Ebner B., Gerlach F., Fuchs C., Hankeln T.: Neuroglobin and Cytoglobin: Genes, Proteins and Evolution. IUBMB Life. 2004, 56, 703-707, doi: 10.1080/ 15216540500037257.

6. Burmester T., Hankeln T.: What Is the Function of Neuroglobin? J. Exp. Biol. 2009, 212, 1423-1428, doi: 10.1242/jeb.000729.

7. Burmester T., Weich B., Reinhardt S., Hankeln T.: A vertebrate globin expressed in the brain. Nature 2000, 407, 6803, 520-523.

8. Chan S., Saraswathy S., Rehak M., Rao N.: Neuroglobin protection in retinal ischemia. Invest. Ophthalmol. Vis. Sci. 2012, 532, 704-711.

9. Costa B., Fawcett R., Li G. Y., Safa N. N.: Orally administered epigallocatechin gallate attenuates slight induced photoreceptor damage. Brain research bulletin 2008, 764, 412-423

10. Ding $G$.: Research status of brain-globin in the retina. Journal of clinical ophthalmology 2010, 186, 573-575.

11. Ding G., Yan Z., He T.: The appearance of white and red eggs in rat retina during the death of acute ischemic ganglion cells. Zhonghua eye science journal 2010, 467, 590-596.

12. Fordel E., Thijs L., Martinet W., Schrijvers D., Moens L., Dewilde S.: Anoxia or oxygen and glucose deprivation in sh-sy5y cells: a step closer to the unraveling of neuroglobin and cytoglobin functions. Gene 2007, 3982, 114-122.

13. Geuens E., Brouns I., Flamez D., Dewilde S., Timmermans J., Moens L.: A globin in the nucleus. J. Biol. Chem. 2003, 278, 30417-30420.

14. Greenberg D., Jin K., Khan A.: Neuroglobin: an endogenous neuroprotectant. Current opinion in pharmacology 2008, 81, 20-24.

15. Li Y., Liu H., Tong Y.: Immunohistochemistry of the distribution of brain globin in rat eyeball. Ophthalmic Research 2000, 245, 46 1-4 4.

16. Nayak G., Prentice M., Milton S.: Role of neuroglobin in regulating reactive oxygen species in the brain of the anoxia-tolerant turtle trachemys scripta. Journal of neurochemistry 2010, 1102, 603-612.

17. Ostojic J., Grozdanic D. S., Syed N. A., Hargrove M. S., Trent J. T., Kuehn M. H., Kwon Y. H., Kardon R. H., Sakaguchi S. D.: Patterns of distribution of oxygen-binding globins, neuroglobin and cytoglobin in the human retina. Arch. Ophthalmol. 2008, 12611, 1530-1536.

18. Ostoji J., Sakaguchi D., De Lathouder Y., Hargrove M. S., Trent J. T., Kwon Y. H., Kardon R. H., Kuehn M. H., Betts D. M., Grozdanic S.: Neuroglobin and cytoglobin: oxygen-binding proteins in retinal neurons. Investigative ophthalmology \& visual science. 2006, 473,1016-1023.

19. Ran J., Sun Q., Bi X.: Expression of Neuroglobin mRNA in rat eyeball. Chongqing med. 2003, 47, 1035-1036.

20. Roberts A., Gaffney E., Luthert P. J., Foss A., Bryne H.: Retinal oxygen distribution and the role of Neuroglobin. Journal of mathematical biology 2016, 731, 1-38.

21. Schmidt M., Giessl A., Laufs T., Hankeln T., Wolfrum U., Burmester T.: How does the eye breath? Evidence for neuroglobin-mediated oxygen supply in the mammalian retina. J. Biol. Chem. 2783, 1932-1935.

22. Shi S., Feng $X$.: Expression of myoglobin in acute retinal ischemic injury in rats. Ophthalmic Research 2009, 2710, 915-918.

23. Wei X., Deng Y., Liu X.: How can photoreceptor cells breathe in the retina? Neuroglobin may be the answer. Neural regeneration research 2010, 59, 720-720

24. Xu Q., Huang F., Wei $Y$.: Subcellular distribution of myoglobin expression in the retina of adult rats. International journal of ophthalmology. 2009, 1, 35-37. (In Chinese with English abstract).

25. Xu Q., Huang X., Wei X.: Ultrastructural study of the intraretinal distribution of neuroglobin proteins in normal adult rats. International journal of ophthalmology. 91, 35-37. (In Chinese)

26. Yu Y., Cringle J.: Oxygen distribution and consumption within the retina in vascularized and avascular retinas and animal models of retinal disease. Prog. Retina Eye Res. 2001, 202, 175-208.

27. Yan G., Yue H., Duan W.: The effect of oxygen on the surface of membraneencephaloglobin in rabbit optic network. New progress in ophthalmology 2010, 307, 618-621.

28. Yang Y., Liu X., Gao X.: Distribution of Neuroglobin in the retina of adult sheep. Chinese Journal of anatomy 2015, 3, 275-278 (In Chinese).

29. Ying Q., Feng Y. Z., Guang H. P., Yu Z., Guang M. W., Wen Z. W., Jing M., Shi $J$. R.: Characteristics and visual outcomes of patients hospitalized for ocular trauma in central China. International Journal of Ophthalmology 2015, 8, $162-168$

Corresponding author: PhD Xia Liu, College of Life Science and Technology, Gansu Agricultural University, Lanzhou City, Gansu Province, People's Republic of China 